\title{
Health Promoting Lifestyle Behaviors and Associated Predictors Among Clinical Nurses in China: A Cross- Sectional Study
}

\author{
Wen Zeng \\ Guizhou Provincial People's Hospital \\ Qian Fang \\ Guizhou Provincial People's Hospital \\ Shan He \\ Guizhou Provincial People's Hospital \\ Qiping An \\ Guizhou Provincial People's Hospital \\ Juan Li ( $\nabla$ juanli0920@126.com ) \\ Guizhou Provincial People's Hospital \\ Yuanrong Yao \\ Guizhou Provincial People's Hospital
}

\section{Research Article}

Keywords: Health Promoting Behaviors, Health Promotion, Nurses' Self-care, Nurses' Health

Posted Date: March 11th, 2021

DOI: https://doi.org/10.21203/rs.3.rs-272290/v1

License: (c) (i) This work is licensed under a Creative Commons Attribution 4.0 International License. Read Full License

Version of Record: A version of this preprint was published at BMC Nursing on November 17th, 2021. See the published version at https://doi.org/10.1186/s12912-021-00752-7. 


\section{Abstract}

Background: Nurses play a core role and encompass the main workforce in health care systems. Their role model of health promoting behaviors would directly or indirectly affect their clients' beliefs or attitudes of health promotion. There is limited evidence on health promoting lifestyle behaviors in clinical registered nurses. To explore the health promoting lifestyle behaviors and associated influencing factors among clinical registered nurses in China.

Methods: A multi-center cross-sectional anonymous online survey was conducted in 2020. Participants were asked to complete social demographic information as well as the revised Chinese edition of Health Promoting Lifestyle Profile. Independent-Sample T Test, One-Way ANOVA and categorical regression (optimal scaling regression) were main methods to analysis the relationship between demographic data and score of health promoting lifestyle behaviors.

Results: 19422 nurses were included in the study. The mean score of self-actualization, health responsibility/physical activity, nutrition, job safety, interpersonal support and overall Health Promoting Lifestyle Profile were, 27.61 \pm 5.42 out of a score of $36,22.71 \pm 7.77$ out of a score of $44,10.43 \pm 2.97$ out of a score of $16,22.05 \pm 3.97$ out of a score of 28 , $20.19 \pm 4.67$ out of a score of 28 , and $102.99 \pm 19.93$ out of a score of 144 , respectively. There was a significant relationship among Hospital levels, working years, nightshift status, and monthly income per person and mean score of all subscales and the overall Health Promoting Lifestyle Profile $(P<0.05)$.

Conclusions: Nurses participated in the study presented moderate level of health promoting lifestyle behaviors. Hospital levels, working years, nightshift status, and monthly income per person were predictors for all subscales and overall HPLP.

\section{Background}

Health is a global theme. More empirical evidence suggest that it is much more important to take individuals' capacity to promote their health into consideration rather than to treat disease [1]. The rapid growing evidence indicate that individuals could take measures to promote their health and well-being [2]. Health Promotion (HP) is one of the core determinants and strategies for individuals to facilitate and maintain their health [3], and it has become to global importance [4]. In terms of HP, it has been defined in various way and the World Health Organization (WHO) defined it as "the process of enabling people to increase control over, and to improve, their health." [5]. Some author suggested that HP should be a guiding philosophy in the health care process [6]. Recent years, there is a great emphasis on Health Promoting Lifestyle Behaviors (HPLB). HPLB, which is one of the key concepts in nursing field [7], is defined as individuals' actions and beliefs on multiple levels of HP including self-actualization, physical activities/health responsibility, food choices, interpersonal support [8] to enhance or preserve the level of well-being or reduce the incidence of illness [9].

Nurses play a core role and encompass the main workforce in health-care systems. According to the statistics of WHO, there are 43.5 million heath workers worldwide, while nurses are more than 20 million [10]. Notably, more than 4 million registered nurses are in China [11]. In other words, Chinese nurses account for nearly one fifth of global nurses. Nurses delivery diverse healthcare services in different settings as health care providers, protectors, communicators, coordinators, decision-makers and teachers [12]. In fact, nurses are usually the first responders to different health-related conditions to promote health, rehabilitation and prevent disease[12]. More important, Nurses usually act as a role model, a leader as well as a HP practitioner for the public in empowering and motivating individuals and communities to engage in HP decision-making, HP action and activities to promote their health condition [3, 13-15]. Empirical evidence show that nurses' own health practices could largely produce an effect on the effectiveness of the health intervention provided for their clients [9]. 
The importance of nurses' role in HP has been largely reported, it is believed that nurses' role model of HP behaviors would directly or indirectly affect their clients' belief or attitude of health promotion [14]. Therefore, it is important to know the level of HPLB in nurses. To our best knowledge, most studies on HPLB focus on nursing students $[8,9,13,14$, 16-18] or patients [19]. There is limited evidence on HPLB in clinical registered nurses. Besides, most measurements used to examine individual HPLB do not contain the aspect of job security. Therefore, a modified Health Promoting Lifestyle Profile (HPLP) would be used to examine the HPLB of clinical nurses in China. Additionally, the current study aimed to explore the associated factors that influence the level of HPLB.

\section{Methods}

\subsection{Design, setting and participants}

A multi-center cross-sectional survey was carried out from January 8th 2020 to January $30^{\text {th }} 2020$ in 42 hospitals from 26 cities, 16 provinces in China. These hospitals, which were representative for different levels of healthcare services, were including primary, secondary and tertiary institutions. Registered nurses working as staff nurses in the selected hospital were included. Nursing students and retired nurses were excluded.

The investigation was completed online. Firstly, researchers imported the informed consent agreement and the questionnaire of the revised Chinese edition of HPLP [20] on an online survey plat, Chinese Questionnaire Star (https://www.wjx.cn/), then freely created a QR code. Researchers released the QR code through a social media application WeChat. Participants could scan the QR to read and submit the informed consent agreement. After that, they could choose to fulfill the questionnaire. Each participant was only allowed to submit once in order to avoid double submission.

\subsection{Variables and assessment}

Independent variables in the study were some sociodemographic characteristics including gender, age, basic education background, highest education background, hospital level, working years, nightshift status, working department, marital status, number of children, living with parents, monthly income per family member.

In order to evaluate the level of nurses' HPLB, a modified Chinese edition of HPLP [20] was used in the study. The instrument, which was originally developed by Walker, Sechrist and Pender [21] and translated into the Chinese edition by Chen et al [22]. Based on the Chinese edition of HPLP, Sun, Huang and Ling [20] developed a modified Chinese edition of HPLP which consists of 5 subscales (38 items), namely, self-actualization ( 9 items), health responsibility/physical activities (11 items), nutrition (4 items), job security ( 7 items) and interpersonal support (7 items). The instrument is a 4 Likert-scale and the score of each item ranges from 1 to 4: $1=$ never, 2=sometimes, 3=often, 4=routinely. The overall score of the HPLP ranges from 38 to 144 . Higher score represents better HPLB. The Cronbach's alpha was 0.9278 , and 3 weeks of test-retest reliability Cronbach's alpha was 0.818 .

\subsection{Data collection}

Two researchers who didn't know the study design collected the data. Before the data collection, those two researchers received training courses about how to collect the data, check the data, input and code the data into IBM SPSS 25.0. After the training courses, they took part in an exam related to the data collection. Only if they passed through the exam could they involved in collecting data.

\subsection{Data analysis}


All data was imported into IBM SPSS 25.0. Mean \pm standard deviation (M $\pm S D$ ) was used to describe the continuous data. Categorical variables were presented as frequency $(\mathrm{N})$ and percentages. To investigate the potential factors associated with HPLP, univariate analysis was conducted first to examine the difference of mean score among groups. At this stage, Independent-Sample T Test and One-Way ANOVA were performed. Secondly, categorical regression (optimal scaling regression) was the main method to analysis factors which had been confirmed to be statistically significant in the univariate analysis in order to test the correlation between the demographic data and HPLP. In the current study, $P$ value was two-tailed and we inferred statistical significance if a was less than 0.05 .

\section{Results}

\section{1 demographic characteristics of the participants}

19522 nurses completed the questionnaires. However, 100 questionnaires were removed from analysis because of following reasons: (1) Responders' age was logically incorrect; (2) Participants' working years were more than their age. Therefore, effective respond rate was $99.49 \%$.

As shown in Table 1, female nurses dominated the main responders (95.66\%). Participants' age ranged from 18 to 62 years old. Most (55.11\%) of the included nurses were with associate degree, while only 4531 nurses (23.33\%) originally graduated from college or university and got bachelor degree. However, $54.91 \%$ of the included nurses finally got bachelor degree after receiving continuous education. 10487 nurses were working at a secondary level of hospital and 8619 nurses were at tertiary hospital. In the current study, working years of nurses ranged from 1 to 41 years. Nurses who worked nightshift were more than $55 \%$. Besides, nurses on the schedule of nightshift working were younger, less working age, mainly never married, less educated than those without nightshift. And more than $70 \%$ of the respondents worked at the Inpatient General Department. $68.02 \%$ of the nurses were married and $28.71 \%$ were never married. Only $36.75 \%$ of the nurses reported without any child. Nearly $70 \%$ of the respondents reported monthly income per family

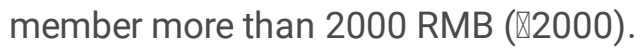




\begin{tabular}{|c|c|c|}
\hline & Frequency & Percentage \\
\hline \multicolumn{3}{|l|}{ Gender } \\
\hline Male & 842 & $4.34 \%$ \\
\hline Female & 18580 & $95.66 \%$ \\
\hline \multicolumn{3}{|l|}{ Age } \\
\hline$\leq 25$ year & 4636.0 & $23.87 \%$ \\
\hline $26 \bowtie 35$ year & 11329.0 & $58.33 \%$ \\
\hline $36 \varangle 45$ year & 2535.0 & $13.05 \%$ \\
\hline$\geq 46$ year & 922.0 & $4.75 \%$ \\
\hline \multicolumn{3}{|l|}{ Basic education level } \\
\hline Secondary diploma & 4187 & $21.56 \%$ \\
\hline Associate degree & 10704 & $55.11 \%$ \\
\hline Baccalaureate degree & 4531 & $23.33 \%$ \\
\hline \multicolumn{3}{|l|}{ Highest education level } \\
\hline Secondary diploma & 495 & $2.55 \%$ \\
\hline Associate degree & 8234 & $42.39 \%$ \\
\hline Baccalaureate degree & 10664 & $54.91 \%$ \\
\hline Master's degree/PHD & 29 & $0.15 \%$ \\
\hline \multicolumn{3}{|l|}{ Hospital level } \\
\hline Primary or community & 316 & $1.63 \%$ \\
\hline Secondary & 10487 & $54.00 \%$ \\
\hline Tertiary & 8619 & $44.38 \%$ \\
\hline \multicolumn{3}{|l|}{ Working years } \\
\hline$\leq 5$ year & 8788 & $45.25 \%$ \\
\hline $6 \otimes 15$ year & 8243 & $42.44 \%$ \\
\hline $16 \llbracket 25$ year & 1523 & $7.84 \%$ \\
\hline$\geq 26$ year & 868 & $4.47 \%$ \\
\hline \multicolumn{3}{|l|}{ Nightshift status } \\
\hline Yes & 10846 & $55.84 \%$ \\
\hline No & 8576 & $44.16 \%$ \\
\hline \multicolumn{3}{|l|}{ Working department } \\
\hline Outpatient & 2277 & $11.72 \%$ \\
\hline
\end{tabular}




\begin{tabular}{|c|c|c|}
\hline Emergency & 1165 & $6.00 \%$ \\
\hline Impatient general ward & 13599 & $70.02 \%$ \\
\hline ICU & 2381 & $12.26 \%$ \\
\hline \multicolumn{3}{|l|}{ Marital status } \\
\hline Single & 5577 & $28.71 \%$ \\
\hline Married & 13211 & $68.02 \%$ \\
\hline Widowed & 47 & $0.24 \%$ \\
\hline Divorced & 587 & $3.02 \%$ \\
\hline \multicolumn{3}{|l|}{ Number of children } \\
\hline 0 & 7138 & $36.75 \%$ \\
\hline 1 & 7701 & $39.65 \%$ \\
\hline 2 & 4507 & $23.21 \%$ \\
\hline 3 & 76 & $0.39 \%$ \\
\hline \multicolumn{3}{|l|}{ Living with parents } \\
\hline Yes & 9852 & $50.73 \%$ \\
\hline No & 9570 & $49.27 \%$ \\
\hline \multicolumn{3}{|l|}{ Per capita income } \\
\hline$\leq 550$ & 690 & $3.55 \%$ \\
\hline $551 \rrbracket 1200$ & 2185 & $11.25 \%$ \\
\hline $1201 \llbracket 2000$ & 3005 & $15.47 \%$ \\
\hline $2001 \varangle 3000$ & 5025 & $25.87 \%$ \\
\hline $3001 \llbracket 6000$ & 6142 & $31.62 \%$ \\
\hline$\nabla 6000$ & 2375 & $12.23 \%$ \\
\hline
\end{tabular}

\subsection{Distribution of mean scores of subscales and overall HPLP}

In the current study, the mean score of self-actualization, health responsibility/physical activity, nutrition, job safety, interpersonal support and overall HPLP were $27.61 \pm 5.42$ out of a score of $36,22.71 \pm 7.77$ out of a score of 44 , $10.43 \pm 2.97$ out of a score of $16,22.05 \pm 3.97$ out of a score of $28,20.19 \pm 4.67$ out of a score of 28 , and $102.99 \pm 19.93$ out of a score of 144 , respectively.

In the process of univariate analysis, all demographic data including gender, age, basic education level, highest education level, hospital level, working years, nightshift status, working department, marital status, number of children, living with parents, monthly income per person were analyzed. The outcome of univariate analysis was presented in Table 2. The mean score of all subscales and the overall HPLP differed in participants with different ages, hospital level, working years, nightshift status, working department, marital status and monthly income per person, and such differences were statistically significant $(P<0.05)$. 
Table 2 also presented horizontal comparison in mean score of 5 subscales and overall HPLP in different demographic characteristics. Regarding nurses' age, we found that participants who aged more than 45 got higher mean score of all 5 subscales and the overall HPLP than other age groups in the study. Such a difference was significant through pairwise comparison $(P<0.001)$. In terms of highest education level shown in Table 2 , the mean score of all subscales and overall HPLP was higher in participants with postgraduate degree or above than that in nurses with other education background. Moreover, such differences in subscales and overall HPLP except in job safety were significant $(P<0.001)$. Additionally, higher mean score was presented in participants working in Tertiary hospitals and the difference was statistically significant when performing pairwise comparison $(P<0.05)$. Moreover, the result showed significant difference in the mean score of all subscales and the overall HPLP in subjects with different working years $(P<0.001)$. As seen in Table 2, nurses who worked more than 26 years reported higher mean score than other groups $(P<0.05)$. Compared with nurses who were not on nightshift rotation, nurses on nightshift rotation got lower mean score in all subscales and the overall HPLP, and the statistical test indicated that such differences were significant $(P<0.001)$. Surprisingly, it was reported that widowed nurses got the highest mean score for each subscale and the overall HPLP in the current study. According to the Table 2, there was a significant difference between the mean score of each dimension of the scale and the working departments participants working in $(P<0.001)$. Not surprisingly, nurses working in the ICU got the lowest mean score while nurses in the Outpatient got the highest mean score. As for monthly income per person, it was found that the higher income, the higher mean score in the study. Obviously, nurses with monthly income per person $₫ 6000$ Yuan got the highest mean score for each dimension and the overall HPLP. 
Table 2. Distribution of Subscales and overall HPLP

\begin{tabular}{|c|c|c|c|c|}
\hline $\begin{array}{l}\text { self- } \\
\text { actualization }\end{array}$ & $\begin{array}{l}\text { health } \\
\text { responsibility / } \\
\text { physical activity }\end{array}$ & Nutrition & job safety & $\begin{array}{l}\text { interpersonal } \\
\text { support }\end{array}$ \\
\hline
\end{tabular}

\section{Gender}

\begin{tabular}{|c|c|c|c|c|c|c|}
\hline Male & $28.00 \pm 5.57$ & $25.09 \pm 8.95$ & $10.31 \pm 2.98$ & $22.41 \pm 4.05$ & $20.65 \pm 4.76$ & $106.45 \pm 21.50$ \\
\hline Female & $27.59 \pm 5.41$ & $22.60 \pm 7.69$ & $10.43 \pm 2.97$ & $22.03 \pm 3.97$ & $20.17 \pm 4.66$ & $102.83 \pm 19.84$ \\
\hline $\mathrm{t}$ & 2.117 & 7.941 & -1.202 & 2.646 & 2.94 & 4.796 \\
\hline$P$ & 0.034 & $\varangle 0.001$ & 0.23 & 0.008 & 0.003 & $\varangle 0.001$ \\
\hline \multicolumn{7}{|l|}{ Age } \\
\hline$\leq 25$ year & $27.11 \pm 5.41$ & $23.16 \pm 7.92$ & $9.92 \pm 2.95$ & $22.23 \pm 3.98$ & $20.64 \pm 4.69$ & $103.05 \pm 20.16$ \\
\hline $26 \bowtie 35$ year & $27.51 \pm 5.44$ & $22.19 \pm 7.77$ & $10.23 \pm 2.95$ & $21.85 \pm 4.00$ & $19.86 \pm 4.69$ & $101.64 \pm 19.95$ \\
\hline $36 \varangle 45$ year & $28.38 \pm 5.20$ & $23.15 \pm 7.42$ & $11.52 \pm 2.74$ & $22.35 \pm 3.85$ & $20.46 \pm 4.51$ & $105.86 \pm 18.94$ \\
\hline$\geq 46$ year & $29.19 \pm 5.18$ & $25.61 \pm 6.99$ & $12.40 \pm 2.50$ & $22.81 \pm 3.84$ & $21.26 \pm 4.31$ & $111.27 \pm 18.34$ \\
\hline $\mathrm{F}$ & 61.446 & 77.314 & 387.735 & 28.877 & 3558.998 & 3561.706 \\
\hline$P$ & $\bowtie 0.001$ & 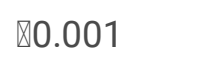 & 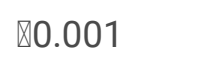 & 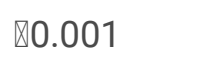 & $\nabla 0.001$ & $凶 0.001$ \\
\hline \multicolumn{7}{|c|}{ Basic education level } \\
\hline $\begin{array}{l}\text { Secondary } \\
\text { diploma }\end{array}$ & $28.00 \pm 5.28$ & $22.80 \pm 7.35$ & $10.81 \pm 2.96$ & $22.07 \pm 3.87$ & $20.16 \pm 4.54$ & $103.84 \pm 19.17$ \\
\hline $\begin{array}{l}\text { Associate } \\
\text { degree }\end{array}$ & $27.35 \pm 5.39$ & $22.60 \pm 7.85$ & $10.19 \pm 2.95$ & $21.96 \pm 4.00$ & $20.12 \pm 4.70$ & $102.22 \pm 20.06$ \\
\hline $\begin{array}{l}\text { Baccalaureate } \\
\text { degree }\end{array}$ & $27.87 \pm 5.56$ & $22.90 \pm 7.95$ & $10.62 \pm 2.99$ & $22.23 \pm 4.00$ & $20.39 \pm 4.71$ & $104.01 \pm 20.24$ \\
\hline $\mathrm{F}$ & 28.817 & 2.756 & 77.963 & 7.28 & 5.124 & 17.614 \\
\hline$P$ & $\varangle 0.001$ & 0.064 & $\triangle 0.001$ & 0.001 & 0.006 & $\triangle 0.001$ \\
\hline
\end{tabular}

Highest education level

\begin{tabular}{|c|c|c|c|c|c|c|}
\hline $\begin{array}{l}\text { Secondary } \\
\text { diploma }\end{array}$ & $27.38 \pm 5.43$ & $23.10 \pm 7.10$ & $10.71 \pm 2.92$ & $21.87 \pm 4.09$ & $20.34 \pm 4.58$ & $103.40 \pm 19.32$ \\
\hline $\begin{array}{l}\text { Associate } \\
\text { degree }\end{array}$ & $27.44 \pm 5.41$ & $23.07 \pm 7.93$ & $10.26 \pm 3.01$ & $22.08 \pm 4.01$ & $20.38 \pm 4.69$ & $103.23 \pm 20.29$ \\
\hline $\begin{array}{l}\text { Baccalaureate } \\
\text { degree }\end{array}$ & $27.74 \pm 5.42$ & $22.41 \pm 7.65$ & $10.54 \pm 2.94$ & $22.03 \pm 3.94$ & $20.04 \pm 4.65$ & $102.76 \pm 19.67$ \\
\hline $\begin{array}{c}\text { Master's } \\
\text { degree/PHD }\end{array}$ & $29.62 \pm 5.49$ & $25.48 \pm 8.82$ & $12.52 \pm 3.00$ & $23.41 \pm 4.39$ & $21.79 \pm 4.74$ & $112.83 \pm 21.44$ \\
\hline $\mathrm{F}$ & 6.355 & 12.31 & 19.947 & 1.715 & 9.712 & 2.961 \\
\hline$P$ & $\varangle 0.001$ & $\varangle 0.001$ & $\varangle 0.001$ & 0.162 & 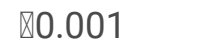 & 0.035 \\
\hline
\end{tabular}




\begin{tabular}{|c|c|c|c|c|c|c|}
\hline $\begin{array}{l}\text { Primary or } \\
\text { community }\end{array}$ & $26.64 \pm 5.68$ & $22.25 \pm 7.77$ & $10.25 \pm 3.05$ & $21.63 \pm 4.11$ & $19.84 \pm 4.87$ & $100.60 \pm 20.67$ \\
\hline Secondary & $27.19 \pm 5.37$ & $22.22 \pm 7.51$ & $10.21 \pm 2.90$ & $21.73 \pm 3.97$ & $19.83 \pm 4.61$ & $101.18 \pm 19.42$ \\
\hline Tertiary & $28.15 \pm 5.41$ & $23.32 \pm 8.03$ & $10.69 \pm 3.03$ & $22.46 \pm 3.94$ & $20.65 \pm 4.69$ & $105.28 \pm 20.28$ \\
\hline $\mathrm{F}$ & 80.004 & 47.695 & 63.286 & 83.569 & 74.579 & 102.561 \\
\hline$P$ & $\llbracket 0.001$ & $\llbracket 0.001$ & $\llbracket 0.001$ & $\bowtie 0.001$ & $\llbracket 0.001$ & $\varangle 0.001$ \\
\hline
\end{tabular}

\section{Working years}

\begin{tabular}{|c|c|c|c|c|c|c|}
\hline$\leq 5$ year & $27.24 \pm 5.42$ & $22.90 \pm 7.90$ & $9.93 \pm 2.94$ & $22.03 \pm 4.05$ & $20.31 \pm 4.71$ & $102.41 \pm 20.18$ \\
\hline $6 \otimes 15$ year & $27.64 \pm 5.42$ & $22.05 \pm 7.67$ & $10.50 \pm 2.93$ & $21.87 \pm 3.94$ & $19.87 \pm 4.66$ & $101.95 \pm 19.75$ \\
\hline $16 \varangle 25$ year & $28.62 \pm 5.24$ & $23.56 \pm 7.43$ & 11.74592 .71 & $22.60 \pm 3.77$ & $20.71 \pm 4.52$ & $107.23 \pm 18.80$ \\
\hline$\geq 26$ year & $29.22 \pm 5.13$ & $25.57 \pm 6.98$ & $12.44 \pm 2.44$ & $22.82 \pm 3.79$ & $21.19 \pm 4.29$ & $111.25 \pm 18.18$ \\
\hline $\mathrm{F}$ & 60.651 & 77.093 & 399.449 & 27.437 & 37.654 & 95.36 \\
\hline$P$ & $\mathbb{\nabla} 0.001$ & $\nabla 0.001$ & $\varangle 0.001$ & $\llbracket 0.001$ & $\varangle 0.001$ & $\varangle 0.001$ \\
\hline
\end{tabular}

Nightshift status

\begin{tabular}{|c|c|c|c|c|c|c|}
\hline Yes & $27.15 \pm 5.45$ & $22.41 \pm 7.87$ & $9.78 \pm 2.91$ & $21.86 \pm 4.01$ & $19.78 \pm 4.70$ & $100.97 \pm 20.11$ \\
\hline No & $28.19 \pm 5.31$ & $23.10 \pm 7.62$ & $11.24 \pm 2.85$ & $22.30 \pm 3.92$ & $20.71 \pm 4.58$ & $105.54 \pm 19.40$ \\
\hline $\mathrm{t}$ & -13.386 & -6.196 & -35.138 & -7.719 & -13.873 & -16.037 \\
\hline$P$ & $\llbracket 0.001$ & $\varangle 0.001$ & $凶 0.001$ & $\otimes 0.001$ & $\varangle 0.001$ & $\varangle 0.001$ \\
\hline
\end{tabular}

Working department

\begin{tabular}{|c|c|c|c|c|c|c|}
\hline Outpatient & $27.98 \pm 5.33$ & $23.42 \pm 7.39$ & $11.39 \pm 2.90$ & $22.40 \pm 3.89$ & $21.02 \pm 4.54$ & $106.20 \pm 19.22$ \\
\hline Emergency & $27.33 \pm 5.37$ & $22.86 \pm 7.80$ & $10.15 \pm 2.91$ & $21.99 \pm 3.90$ & $19.90 \pm 4.50$ & $102.23 \pm 19.60$ \\
\hline $\begin{array}{l}\text { Impatient } \\
\text { general ward }\end{array}$ & $27.61 \pm 5.45$ & $22.65 \pm 7.79$ & $10.38 \pm 2.96$ & $22.01 \pm 4.01$ & $20.18 \pm 4.70$ & $102.82 \pm 20.06$ \\
\hline ICU & $27.38 \pm 5,30$ & $22.33 \pm 7.90$ & $9.87 \pm 2.91$ & $22.00 \pm 3.85$ & $19.64 \pm 4.57$ & $101.22 \pm 19.71$ \\
\hline $\mathrm{F}$ & 6.015 & 69.188 & 117.691 & 6.83 & 38.431 & 26.975 \\
\hline$P$ & $\triangle 0.001$ & $\llbracket 0.001$ & 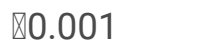 & $\nabla 0.001$ & $\otimes 0.001$ & $\otimes 0.001$ \\
\hline \multicolumn{7}{|l|}{ Marital status } \\
\hline Single & $27.13 \pm 5.44$ & $23.15 \pm 7.88$ & $9.88 \pm 2.92$ & $22.27 \pm 3.94$ & $20.57 \pm 4.65$ & $102.99 \pm 20.08$ \\
\hline Married & $27.78 \pm 5.38$ & $22.50 \pm 7.71$ & $10.65 \pm 2.96$ & $21.94 \pm 3.99$ & $20.01 \pm 4.67$ & $102.88 \pm 19.85$ \\
\hline Widowed & $28.89 \pm 5.13$ & $26.47 \pm 7.92$ & $11.43 \pm 2.83$ & $23.21 \pm 3.11$ & $21.91 \pm 3.80$ & $111.89 \pm 18.16$ \\
\hline Divorced & $28.09 \pm 5.59$ & $23.06 \pm 7.69$ & $10.60 \pm 3.10$ & 22.463 .96 & $20.51 \pm 4.67$ & $104.74 \pm 20.28$ \\
\hline $\mathrm{F}$ & 21.565 & 13.167 & 92.192 & 12.479 & 22.057 & 4.772 \\
\hline$P$ & $\bowtie 0.001$ & 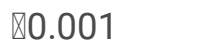 & 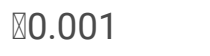 & $\nabla 0.001$ & $\otimes 0.001$ & $\otimes 0.001$ \\
\hline
\end{tabular}

Number of children 


\begin{tabular}{|c|c|c|c|c|c|c|}
\hline 0 & $27.20 \pm 5.44$ & $23.07 \pm 7.90$ & $9.93 \pm 2.93$ & $22.19 \pm 3.97$ & $20.55 \pm 4.65$ & $102.94 \pm 20.11$ \\
\hline 1 & $27.81 \pm 5.43$ & $22.65 \pm 7.68$ & $10.69 \pm 2.98$ & $22.06 \pm 3.95$ & $20.09 \pm 4.64$ & $103.29 \pm 19.82$ \\
\hline 2 & $27.90 \pm 5.32$ & $22.26 \pm 7.66$ & $10.76 \pm 2.92$ & $21.83 \pm 4.02$ & $19.80 \pm 4.71$ & $102.54 \pm 19.78$ \\
\hline 3 & $28.79 \pm 5.19$ & $22.13 \pm 8.68$ & $10.83 \pm 3.05$ & $21.55 \pm 4.11$ & $19.82 \pm 5.01$ & $103.11 \pm 22.11$ \\
\hline $\mathrm{F}$ & 22.913 & 10.438 & 106.93 & 8.016 & 26.651 & 1.38 \\
\hline$P$ & $₫ 0.001$ & $\$ 0.001$ & $₫ 0.001$ & $\otimes 0.001$ & $₫ 0.001$ & 0.247 \\
\hline \multicolumn{7}{|c|}{ Living with parents } \\
\hline Yes & $27.62 \pm 5.43$ & $22.58 \pm 7.81$ & $10.46 \pm 2.97$ & $22.02 \pm 4.00$ & $20.18 \pm 4.69$ & $102.86 \pm 19.97$ \\
\hline No & $27.59 \pm 5.41$ & $22.84 \pm 7.71$ & $10.39 \pm 2.97$ & $22.08 \pm 3.95$ & $20.21 \pm 4.64$ & $103.12 \pm 19.89$ \\
\hline $\mathrm{t}$ & 0.357 & -2.32 & 1.463 & -0.939 & -0.495 & -0.892 \\
\hline$P$ & 0.721 & 0.02 & 0.143 & 0.348 & 0.621 & 0.372 \\
\hline \multicolumn{7}{|c|}{ Monthly income per person } \\
\hline$\leq 550$ & $26.93 \pm 5.98$ & $22.63 \pm 8.34$ & $9.78 \pm 3.12$ & $21.29 \pm 4.33$ & $19.60 \pm 4.91$ & $100.23 \pm 21.76$ \\
\hline $551 \otimes 1200$ & $27.00 \pm 5.45$ & $22.65 \pm 7.80$ & $9.90 \pm 2.93$ & $21.57 \pm 4.03$ & $19.69 \pm 4.72$ & $100.81 \pm 20.16$ \\
\hline $1201 \otimes 2000$ & $27.37 \pm 5.28$ & $22.43 \pm 7.60$ & $10.09 \pm 2.85$ & $21.84 \pm 3.92$ & $19.83 \pm 4.64$ & $101.57 \pm 19.57$ \\
\hline $2001 \rrbracket 3000$ & $27.40 \pm 5.38$ & $22.60 \pm 7.83$ & $10.26 \pm 2.98$ & $21.96 \pm 3.97$ & $20.04 \pm 4.66$ & $102.26 \pm 19.96$ \\
\hline $3001 \rrbracket 6000$ & $27.78 \pm 5.38$ & $22.61 \pm 7.62$ & $10.62 \pm 2.94$ & $22.16 \pm 3.95$ & $20.34 \pm 4.60$ & $103.50 \pm 19.66$ \\
\hline$\$ 6000$ & $28.61 \pm 5.39$ & $23.64 \pm 7.94$ & $11.35 \pm 2.94$ & $22.91 \pm 3.98$ & $21.24 \pm 4.60$ & $107.82 \pm 19.43$ \\
\hline $\mathrm{F}$ & 29.921 & 7.782 & 83.489 & 37.849 & 37.731 & 42.01 \\
\hline$P$ & $\otimes 0.001$ & $₫ 0.001$ & $\otimes 0.001$ & $\otimes 0.001$ & $\otimes 0.001$ & $\otimes 0.001$ \\
\hline
\end{tabular}

\subsection{Results of Category Regression}

In the process, all significant factors in the univariate analysis were included into the category regression in order to determine the correlation of the demographic data and the HPLP. The results were presented in Table 3. It was observed that gender, highest education level, hospital level, working years, nightshift status, number of children and monthly income per person were included into the predicting equation of average score of self-actualization, and the equation was significant (Adjust $\mathrm{R}^{2}=0.023, P<0.001$ ). And in those above factors, hospital level, nightshift status, monthly income per person, working years were the four most important factor which might impact on the mean score of self-

actualization with the importance rate, namely, $27.5 \%, 25.6 \%, 18 \%$ and $13.4 \%$. As for responsibility/physical activity, the predicting equation was also significant (Adjust $R^{2}=0.025, P<0.001$ ), in which all factors significant in the univariate analysis but living with parents were included. And age was the most important factors with the importance rate $31.2 \%$ that might impact the mean score of health responsibility/physical activity. In regard to Nutrition, all included factors significant in the univariate analysis were significant in the predicting equation for the mean score of nutrition (Adjust $\mathrm{R}^{2}=0.093, P<0.001$ ), and nightshift status was found to be the most important factor with the important weight of 42.1\%. Regarding the mean score of job safety, factors including gender, hospital level, working years, nightshift status, working department, marital status, number of children and monthly income per person were included into the predicting equation, and such an equation was tested statistically significant (Adjust $R^{2}=0.024, P<0.001$ ). In such an equation, 
obviously, monthly income per person and hospital level were found as the two most important predicting factor for mean score of job safety. When exploring the predicting model for the mean score of interpersonal support, all included factors but working department were significant (Adjust $\mathrm{R}^{2}=0.042, P<0.001$ ). As shown in Table 3 , it is evident that nightshift status (Importance weight: 24\%) and monthly income per person (Importance weight: 20.5\%) were the two most important predictive factors for interpersonal support. With regard to the total score of HPLP, gender, age, basic education level, highest education level, hospital level, working years, nightshift status, working department, marital status and monthly income per person were predictive variables in the significant equation (Adjust $\mathrm{R}^{2}=0.039, P<0.001$ ), and the results indicated that nightshift status (25.6\%), hospital level (22.2\%), working years (21.8\%) and monthly income per person (17.9\%) were the four most important predictive factors. 
Table 3. Outcome of Category Regression $\beta$ (Importance)

\begin{tabular}{|c|c|c|c|c|c|c|}
\hline & $\begin{array}{l}\text { self- } \\
\text { actualization }\end{array}$ & $\begin{array}{l}\text { health } \\
\text { responsibility } \\
\text { and physical } \\
\text { activity }\end{array}$ & Nutrition & job safety & $\begin{array}{l}\text { interpersonal } \\
\text { support }\end{array}$ & Overall HPLP \\
\hline Gender & $0.025(1.6 \%)$ & $-0.066(16.7 \%)$ & $\longrightarrow$ & $\underset{\star}{0.015(1.1 \%)}$ & $0.021(1.0 \%)$ * & $\underset{\star}{0.043(3.9 \%)}$ \\
\hline Age & $0.018(6.7 \%)$ & $0.092(31.2 \%)$ * & $0.076(17.5 \%)$ * & $-0.036(3.6 \%)$ & $\begin{array}{l}-0.047(5.9 \%) \\
\star\end{array}$ & $\begin{array}{l}-0.031(0.1 \%) \\
\star\end{array}$ \\
\hline $\begin{array}{l}\text { Basic } \\
\text { education } \\
\text { level }\end{array}$ & $0.013(1.4 \%)$ & $\longrightarrow$ & $\begin{array}{l}0.049(-0.007 \%) \\
\star\end{array}$ & $0.015(0.6 \%)$ & $0.040(1.8 \%)$ * & $\begin{array}{l}0.041(1.6 \%) \\
\star\end{array}$ \\
\hline $\begin{array}{l}\text { Highest } \\
\text { education } \\
\text { level }\end{array}$ & $0.013(0.7)$ * & $-0.037(6.0 \%)$ * & $-0.024(-1.1 \%)$ * & $\longrightarrow$ & $\begin{array}{l}-0.059(4.9 \%) \\
\star\end{array}$ & $\begin{array}{l}-0.046(1.3 \%) \\
\star\end{array}$ \\
\hline $\begin{array}{l}\text { Hospital } \\
\text { level }\end{array}$ & $0.073(27.5 \%)$ & $0.068(18.8 \%)$ * & $0.052(4.5 \%)$ * & $0.079(28.4 \%)$ & $0.082(16.5 \%)$ & $0.089(22.2 \%)$ \\
\hline $\begin{array}{l}\text { Working } \\
\text { years }\end{array}$ & $0.037(13.4)$ * & $-0.043(3.6 \%)$ * & $0.079(18.1 \%)$ * & $0.042(9.8 \%)$ & $0.031(4.0 \%)$ * & $\begin{array}{l}0.080(21.8 \%) \\
\star\end{array}$ \\
\hline $\begin{array}{l}\text { Nightshift } \\
\text { status }\end{array}$ & $0.064(25.6 \%)$ & $0.042(7.3 \%)$ * & $0.162(42.1 \%)$ * & $0.051(11 \%)$ * & $\underset{\star}{0.105(24.0 \%)}$ & $0.092(25.6 \%)$ \\
\hline $\begin{array}{l}\text { Working } \\
\text { department }\end{array}$ & $\underset{*}{0.015(0.5 \%)}$ & $-0.023(2.8 \%)$ * & $-0.056(7.5 \%)$ * & $0.014\left(1.7 \% \rrbracket^{\star}\right.$ & $-0.048(8.1 \%)$ & $\begin{array}{l}-0.033(4.6 \%) \\
\text { * }\end{array}$ \\
\hline $\begin{array}{l}\text { Marital } \\
\text { status }\end{array}$ & $0.009(1.9 \%)$ & $-0.024(4.1 \%)$ * & $0.017(0.7 \%)$ * & $\underset{*}{0.053(8.7 \%)}$ & $0.027(3.4 \%)$ * & ${ }_{*}^{0.035}(1.0 \%)$ \\
\hline $\begin{array}{l}\text { Number of } \\
\text { children }\end{array}$ & $0.020(2.7 \%)$ & $-0.035(4.8 \%)$ * & $0.019(1.3 \%)$ * & $-0.016(2.2 \%)$ & $\begin{array}{l}-0.069(10.0 \%) \\
*\end{array}$ & - \\
\hline $\begin{array}{l}\text { Living with } \\
\text { parents }\end{array}$ & $\longrightarrow$ & $0.013(-0.008 \%)$ & $\longrightarrow$ & $\longrightarrow$ & $\longrightarrow$ & $\longrightarrow$ \\
\hline $\begin{array}{l}\text { Monthly } \\
\text { income per } \\
\text { person }\end{array}$ & $0.05(18 \%)$ * & $0.033(5.8 \%)$ * & $0.067(10.1 \%)$ * & $0.087(32.9 \%)$ & $0.091(20.5 \%)$ & $\underset{*}{0.072(17.9 \%)}$ \\
\hline $\mathbf{R}$ & 0.155 & 0.16 & 0.306 & 0.16 & 0.208 & 0.203 \\
\hline $\mathrm{R}^{2}$ & 0.024 & 0.026 & 0.094 & 0.026 & 0.043 & 0.041 \\
\hline Adjust $\mathrm{R}^{2}$ & 0.023 & 0.025 & 0.093 & 0.024 & 0.042 & 0.04 \\
\hline$P$ & $\llbracket 0.001$ & $\varangle 0.001$ & 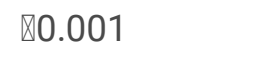 & $\otimes 0.001$ & 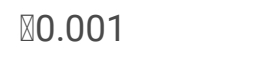 & $\otimes 0.001$ \\
\hline
\end{tabular}

\section{Discussions}

In recent years, HPLB continue to attract researchers' attention. Nevertheless, there is limited evidence on HP in clinical nurses even though they are in a unique position in the healthcare system. Thus, our current study was designed to examine the HPLB and to explore some relative predictors for HPLB in clinical nurses in China. 
In the current study, the mean score of self-actualization, health responsibility/physical activity, nutrition, job safety, interpersonal support and overall HPLP were, respectively, 27.61 $\pm 5.42,22.71 \pm 7.77,10.43 \pm 2.97,22.05 \pm 3.97,20.19 \pm 4.67$ and $102.99 \pm 19.93$, which indicate a moderate level of health promoting behaviors in Chinese clinical nurse. Several previous studies $[8,9,13,14,17,18]$ have examined HPLB in nursing students, and their results were not consistent with our study. On one hand, the subjects we assessed were different. On the other hand, measurements used in those studies differed from that used in the current study. For example, clinical nurses are the ones who offer healthcare for their clients directly while most of nursing students do not. The measurement used in our study includes a subscale of job safety. For those reasons, the findings in the current study are different with previous studies.

There is ongoing debate on the predictors for the HPLB. Some previous studies found that there was a significant correlation between factors such as age, gender, education background and HPLB [2, 16], which was in line with our findings in some degree. Conner and Norman [2] believed that subjects with younger age, better education background usually performed better HPLB. However, our findings indicate that nurses with older age, especially nurses whose age was more than 45 years-old, got the highest average score of HPLP, which was also accentuated by Thacker et al [23]. We suppose that older nurses experience more health-related issues both on patients and on their own, so that they gain a deeper understanding and care more about health. Therefore, they would like to practice more healthier lifestyle behaviors. In accordance with the results of Conner and Norman [2], our study provides additional support for the relationship between education background the health promoting behaviors. Our findings point to the importance of higher education in score of self-actualization, health responsibility, nutrition, interpersonal support and overall HPLP. Specifically, nurses with Master degree/PHD got better performance in those above subscales.

It should be noted that nightshift status and monthly income per family member were also proved as predictors of HPLP in the current study. The current study confirms that nightshift status was the most important predictor of selfactualization, nutrition, interpersonal support and overall HPLP. Additionally, nurses on nightshift rotation got less score than those not on nightshift rotation in all subscales and overall HPLP. In China, most nurses on nightshift rotation works in 3 shifts: $8 \mathrm{am}-4 \mathrm{pm}, 4 \mathrm{pm}-12 \mathrm{mn}, 12 \mathrm{mn}-8 \mathrm{am}$. In addition, nurses have at least 2 days of shift working in a week. Such shifts could offer continuous care services for patients. Unfortunately, internal circadian rhythms are disrupted by those irregular working hours, which leads to sleep disorders, irregular eating habits, irregular exercise or less motivation/empowerment to make healthier lifestyle plan [24]. Even worse, physical and mental tiredness due to nightshift rotation would directly or indirectly increase the consumption of cigarettes or alcohols or junk food [24]. Nurses in a qualitative study stated that irregular rotating shift work made it difficult to participate in a healthy lifestyle behavior because they had to spend time in recovering from such irregular rotation working schedule. As a result, they had less time or energy to hang out with family/friends or to participate to exercise schedule or other stress reduction behaviors $[25,26]$. In terms of monthly income per person, we found that nurses with higher income, they got higher score in all subscales and overall HPLP. Especially, nurses whose monthly income per family member was more than 6000 Yuan got the highest score in each subscale and the overall HPLP, which is consistent with the study by Wang [27]. Multiple factors analysis suggests that monthly income per family member is one of the most important determents of the score of all subscales and overall HPLP. It's possibly because that nurses with higher income would have more chances to behave healthier such as buying quality healthy food, exercising in the gym [27].

Notably, the current study points out the importance of hospital level in predicting the score of subscales and the overall HPLP. Contrary to the results of Wang [27], we surprisingly found that nurses working in the tertiary hospital got the highest mean score in all subscales and the overall HJPLP. Besides, the multiple factors analysis indicates that hospital level was one of the most important predictors for all subscales and the overall HPLP. In China, hospitals are classified into 3 levels including primary level, secondary level and tertiary level. The higher level of a hospital, the wider and more comprehensive health care services will be delivered into the patients [28]. Thus, nurses in the tertiary hospitals usually with higher education background. Although evidence showed that nurses in the tertiary hospitals faces with much

Page 13/17 
higher level of workload [29,30] in China because patients preferred seeking healthcare at higher level hospitals [31]. However, empirical evidence showed that the resilience level of nurses in the tertiary hospitals was much higher than nurses in other level of hospitals due to higher level of welfare as well as more opportunities for continuing education [32]. In general, nurses in the tertiary hospitals get higher payment, higher education background and higher level of resilience and they would cope work-related stress in a positive way [32]. As a result, they might have better understanding about health and have more ways to conduct healthy lifestyle behaviors.

\section{Limitations of the Study}

The main limitation of the study is the design of cross-sectional study. Due to the of the design, we could only access the data at one point in time. Besides, we could we could not gain more and further data, especially about nurses' feeling or experience on HPLB because of lacking of qualitative evidence in this study. Mixed methodology could be used in future studies to take deeper insight of nurses' HPLB. Moreover, bias might occur when participants complete such a self-reporting questionnaire.

\section{Conclusions}

The study aimed to examine the HPLB of Chinese registered nurses and explore relative predictors. The results indicate that nurses participated in the study presented moderate level of all subscales and overall HPLP. Hospital levels, working years, nightshift status, and monthly income per person were predictors for all subscales and overall HPLP.

\section{Abbreviations}

Health Promotion: HP

World Health Organization: WHO

Health Promoting Lifestyle Behaviors: HPLB

Health Promoting Lifestyle Profile: HPLP

Mean \pm standard deviation: $\mathrm{M} \pm \mathrm{SD}$

\section{Declarations}

\section{Ethical Approval and Consent to Participate}

Under the guidance of principles of World Medical Association Declaration of Helsinki [33], it was taken into the first consideration to respect participants' right and to protect their health and rights. The investigation was an anonymous survey. Informed consent was delivered in verbal when participants were included and accessed online. In addition, the informed consent agreement was imported into the survey plat. Subjects agreed the verbal informed consent and could choose to answer the questionnaire based on their willingness. Since the current study was a cross-sectional study in which the only risk was about participants' privacy. Therefore, when conducting the survey, the participants would be informed about the purpose of the study, the way and the time of conducting the survey, their rights to refuse the survey and how their information was stored, protected and used in an academic way. The study was approved by the Ethical Committee of the corresponding institution $₫$ Guizhou Provincial People's Hospital (reference 2018072).

\section{Consent for publication}

Page $14 / 17$ 
Not applicable

\section{Availability of data and materials}

All the data supporting the study findings are within the manuscript. Additional detailed information and raw data are available from the corresponding author on reasonable request.

\section{Competing Interest}

None declared.

\section{Funding}

This study presents independent research funded by National Natural Science Foundation of Guizhou Province (NNSF) (81860245), which offered support in the process of study design and financial support for publication.

\section{Authors' Contribution}

WZ, QF, JL and YY conceived and designed the study. QF, SH and QA collected, input and checked the data. WZ analyzed the data and draft the manuscript. All authors read and approved the final manuscript.

\section{Acknowledgements}

The authors express their sincere thanks to the NNSF, and we appreciate the efforts of all the hospital administrators and Donglan Ling, Chunmei Lv, Xueyan Liu, Xiao Xiao, Yao Liao, Hua Yang, Lianlian Tang, Wenjie Yao, who facilitated the survey. Specially, we express our sincere thanks to Shiyuan Dai, Xiao Hu for their help in analyzing the data. The authors also wish to acknowledge all the nurses who participated in the study.

\section{References}

1. Raingruber B. Contemporary health promotion in nursing practice 2nd [M]: Jones \& Bartlett Publishers; 2017.

2. Conner M, Norman P. Predicting health behaviour[M]: McGraw-Hill Education (UK); 2005.

3. Rahayu E, Setiyani R, Sumarwati M, et al. POSITIVE CORRELATION BETWEN SELF EFFICACY AND HEALTH PROMOTING LIFESTYLE BEHAVIOR OF STUDENTS OFNURSING DEPARTMENT OF JENDERAL SOEDIRMAN UNIVERSITY[J]. Health. 2019;22(11):S340.

4. Phillips A. Effective approaches to health promotion in nursing practice[J]. Nursing Standard. 2019.

5. World Health Organization. The 1st International Conference on Health Promotion, Ottawa, 19861986 [cited 2020 17 December]. Available from: https://www.who.int/teams/health-promotion/enhanced-wellbeing/first-globalconference.

6. Wilson-Barnett J, editor. Health promotion and nursing practice[M]: Blackwell Scientific Publ; 1993.

7. Hosseini M, Sarbakhsh P, Mollaei S. The Relationship between the Family Functions and Health-Promoting Behaviors of Nursing Students in Tehran, Iran[J]. World Family Medicine Journal: Incorporating the Middle East Journal of Family Medicine. 2018;99(5897):1-4.

8. Nassar OS, Shaheen AM. Health-promoting behaviours of university nursing students in Jordan[J]. Health. 2014;6(19):2756.

9. Mak YW, Kao AHF, Tam LWY, et al. Health-promoting lifestyle and quality of life among Chinese nursing students[J]. Primary health care research \& development. 2018;19(6):629-636. 
10. World Health Organization. Nursing and midwifery 2020 [cited 2020 March $4^{\text {th }}$ ]. Available from: https://www.who.int/hrh/nursing_midwifery/en/.

11. Zhang L. Vice premier stresses quality nursing services[N]. People's Daily. 2019.

12. World Health Organization. Global strategic directions for strengthening nursing and midwifery 2016-2020[J]. Geneva: WHO. 2017.

13. Borle PS, Parande M, Tapare V, et al. Health-promoting lifestyle behaviours of nursing students of a tertiary care institute[J]. Int J Community Med Public Health. 2017;44(5):1768-1773.

14. Hong J, Sersri S, Keiwkarnka B. Health-promoting lifestyles of nursing students in Mahidol University[J]. Journal of Public Health and Development. 2007;5(1):27-40.

15. Kemppainen $\mathrm{V}$, Tossavainen $\mathrm{K}$, Turunen $\mathrm{H}$. Nurses' roles in health promotion practice: an integrative review[J]. Health promotion international. 2013;28(4):490-501.

16. Paudel S, GC KB, Bhandari $L$, et al. Health related lifestyle behaviors among undergraduate medical students in Patan Academy of Health Sciences in Nepal[J]. Journal of Biosciences and Medicines. 2017;5(9):43-53.

17. Lee H-J. Health-promoting Lifestyle of Nursing Students: Using Mixed Methods Research[J]. Journal of Korean Academy of Community Health Nursing. 2019;30(4):414-425.

18. Alzahrani SH, Malik AA, Bashawri J, et al. Health-promoting lifestyle profile and associated factors among medical students in a Saudi university[J]. SAGE open medicine. 2019;7:2050312119838426.

19. Tabrizi FM. Health promoting behavior and influencing factors in Iranian breast cancer survivors[J]. Asian Pacific journal of cancer prevention : APJCP. 2015;16(5):1729-1736.

20. Sung S, Huang J, Ling L. Health-Promoting Life Styles and Its Related Factors among Clinical Nurses and Student Nurses[J]. Rong Zong Nursing. 2001;18(2):147-158.

21. Walker SN, Sechrist KR, Pender NJ. The health-promoting lifestyle profile: development and psychometric characteristics[J]. Nursing research. 1987.

22. Chen M, Liao Z, Liao Z, et al. The Preliminary Study of Health-Promotion Life-style of Public Health Nurses[J]. Nursing research. 1994;2(1):41-54.

23. Thacker K, Haas Stavarski D, Brancato V, et al. CE: Original Research: An Investigation into the Health-Promoting Lifestyle Practices of RNs[J]. Am J Nurs. 2016;116(4):24-30; quiz 31.

24. Nea FM, Pourshahidi LK, Kearney J, et al. A Qualitative Exploration of the Shift Work Experience: The Perceived Barriers and Facilitators to a Healthier Lifestyle and the Role of the Workplace Environment[J]. Journal of Occupational \& Environmental Medicine. 2017(No.12):1153-1160.

25. Ross A, Yang L, Wehrlen L, et al. Nurses and health-promoting self-care: Do we practice what we preach?[J]. Journal of nursing management. 2019;27(3):599-608.

26. Cho H, Han K. Associations among nursing work environment and health-promoting behaviors of nurses and nursing performance quality: A multilevel modeling approach[J]. Journal of Nursing Scholarship. 2018;50(4):403410.

27. Wang Z. Health-promoting Behaviors of Nurses and Its Relevant Factors in Grade II and III Hospital of Zhengzhou[D]. Zhengzhou Zhengzhou University; 2020.

28. Cai M, Liu E, Tao H, et al. Does level of hospital matter? A study of mortality of acute myocardial infarction patients in Shanxi, China[J]. American Journal of Medical Quality. 2018;33(2):185-192.

29. Chen X, Ma J, Wu Y, et al. Current status and trend of workload of health professionals in Chinese public hospitals[J]. Journal of Chineses Research Hospitals. 2020;7(1):31-35+141-147. 
30. Liang L-I. Study on the status of nurses' mental workload and its relationship with psychological capital and coping style based on LPA[D]. Shanghai: The Second Military Medical University; 2016.

31. Yip W, Hsiao WC. What drove the cycles of Chinese health system reforms?[J]. Health Systems \& Reform. 2015;1(1):52-61.

32. Chen X, Han B, Xu F, et al. Status of nurses' resilience in China and its influecing factors[J]. Chinese Nursing Management. 2020;20(12):1845-1849.

33. General Assembly of the World Medical Association. World Medical Association Declaration of Helsinki: ethical principles for medical research involving human subjects[J]. J Am Med Assoc. 2013;310(20):2191-2194.

\section{Supplementary Files}

This is a list of supplementary files associated with this preprint. Click to download.

- HPLPScale.docx 